\title{
Des Minguettes à Vaulx-en-Velin : les réponses des pouvoirs publics aux violences urbaines
}

\section{Virginie Linhart}

\section{(2) OpenEdition}

\section{Journals}

Édition électronique

URL : http://journals.openedition.org/conflits/2019

DOI : 10.4000/conflits.2019

ISSN : $1777-5345$

Éditeur :

CCLS - Centre d'études sur les conflits lilberté et sécurité, L'Harmattan

Édition imprimée

Date de publication : 16 juillet 1992

ISSN : 1157-996X

Référence électronique

Virginie Linhart, «Des Minguettes à Vaulx-en-Velin : les réponses des pouvoirs publics aux violences urbaines », Cultures \& Conflits [En ligne], 06 | été 1992, mis en ligne le 31 mars 2006, consulté le 30 mars 2021. URL : http://journals.openedition.org/conflits/2019; DOI : https://doi.org/10.4000/ conflits.2019

Ce document a été généré automatiquement le 30 mars 2021.

Creative Commons License 


\title{
Des Minguettes à Vaulx-en-Velin : les réponses des pouvoirs publics aux violences urbaines
}

\author{
Virginie Linhart
}

1 Il faut revenir à l'événement inaugural que constitue "l'été chaud" des Minguettes de 1981 pour retracer la genèse d'un nouveau raisonnement en matière d'action sociale et urbaine propre à la politique de la ville telle qu'on la définit aujourd'hui. La prise de conscience du malaise des banlieues, qu'inscrivent dans l'espace public les jeunes des cités se livrant à des rodéos de voitures volées et s'opposant aux forces de l'ordre de façon violente, semble favoriser l'émergence de nouvelles formes d'intervention de l'État dans l'espace urbain. A l'occasion de ces incidents, l'ensemble du pays "découvre" l'existence de ces cités à l'abandon tandis que la puissance publique prend acte de la faillite de ses services (école, formation, culture, logement...) inaptes à l'insertion des jeunes en banlieues. Le changement de majorité intervenu en mai 1981, conjugué au constat d'échec et au sentiment d'urgence, a favorisé la mise en place d'une politique innovante laissant une large place à l'expérimentation.

La ville, vecteur de modernisation de l'État?

3 Trois types d'approches sont à l'origine d'une politique de la ville comprise comme la stratégie d'intervention de l'état s'attachant à traiter les problèmes sociaux soigneusement localisés dans des zones de ségrégation sociospaciales du cadre urbain. Une approche institutionnelle d'un classicisme éprouvé d'abord, qui voit la désignation de trois personnalités chargées de rédiger des rapports sur un terrain vierge d'analyse politico-administrative : la ville. Ces travaux $\left({ }^{1}\right)$, commandés par le Premier ministre Pierre Mauroy, vont à n'en pas douter bénéficier de la conjoncture tant dans leur capacité de critique que de proposition; ils sont à l'origine des principales sources d'inspiration de la politique de la ville des dix années à venir ${ }^{2}$. Une deuxième approche baptisée " opération anti-été chaud ", bien plus innovante, réside dans l'organisation ponctuelle et multisectorielle mise en place par la puissance publique aux Minguettes. 
Sous cette appellation, on assiste à une intervention administrative exceptionnelle dans son mode de fonctionnement qui repose sur le partenariat de l'ensemble des services intéressés (Affaires sociales, Equipement, Intérieur) et mobilise des acteurs nouveaux ; ainsi, l'armée prête ses tentes pour que les jeunes des Minguettes partent camper. La troisième approche met à jour la nécessité d'une rénovation du mode d'intervention des services publics. Les missions Dubedout, Schwartz, Bonnemaison qui vont s'atteler à cette tâche ont pour philosophie implicite la modernisation de l'État, au nom d'une démarche transversale et d'une approche globale des problèmes issues du constat suivant : face à une population qui cumule grand nombre de handicaps, si l'on traite chacun d'eux de façon séparée et sans tenir compte du lien de cause à effet de ces problèmes, ils se renforceront mutuellement. Quelques principes novateurs constituent le fondement de la politique de la ville, notamment explicités par le rapport Dubedout ; la notion de projet qui dépasse l'idée stricte de réparation et rejoint celle de développement impliquant l'intervention active des habitants, l'action globale et transversale qui exige de l'ensemble des administrations et de leurs services une adaptation à la réalité sociale avec la même attention pour chacune des prestations (dans les quartiers défavorisés, l'éducation est aussi importante que les transports, que la santé...) et implique une vision non pas catégorielle (un élu pour le logement, un élu pour la culture...) mais territoriale (sur chacun des quartiers, les institutions s'adaptent à la situation et offrent un service "inégalement positif"). A un titre ou à un autre de cette inspiration, procèdent des dispositifs, mis en place en 1982-1983, tels que les Zones d'éducation prioritaires destinées à développer des programmes adaptés aux enfants en difficulté scolaire( $\left.{ }^{3}\right)$, les Missions locales pour l'insertion sociale et professionnelle des jeunes en difficulté, les Conseils communaux de prévention de la délinquance, la mission Banlieues 89 chargée de "faire participer pleinement les banlieues à la civilisation urbaine " et la Commission nationale de développement social des quartiers. Ce sont les impératifs de globalité et de transversalité qui expliquent que ces missions et commissions aient été rattachées au Premier ministre ; celles-ci sont alors définies comme les leviers des différents ministères concernés par une intervention dans le champ urbain. En ce sens, elles représentent bien les instruments d'une modernisation du mode de fonctionnement de l'État.

4 La politique de développement social des quartiers, expérimentale, n'en est pas pour autant marginale : elle a concerné 1,5 million d'habitants résidant dans 350000 logements, dont la moitié devait être réhabilitée ; dans certaines villes, cette procédure a pu toucher une forte proportion des habitants (de $40 \%$ à $60 \%$ de la population communale pour une quinzaine d'entre elles). En outre, elle a mobilisé une dizaine de ministères différents. Dès son origine, cette politique des quartiers est une politique de la ville puisqu'elle s'inscrit dans le cadre de la mise en application des lois de décentralisation de mars $1982^{4}$ et comporte, à travers la procédure des contrats signés, la mise en place d'un système relationnel entre l'État et les élus locaux sur la question de la ville. Dès cette époque, les fonctionnaires du ministère de l'Équipement ${ }^{5}$ ont conscience que l'administration centrale ne sera plus en situation de diriger directement les opérations sur la ville. II n'empêche que le jeu des compétences réparties par la décentralisation ne signifie nullement que les élus seront à même de gérer l'ensemble des problèmes de leur territoire. La nécessité d'outils politiques qui fassent le lien entre l'autonomie locale et les intentions définies à l'échelle nationale (solidarité et innovation) apparait alors évidente. C'est sur ce lien problématique entre cette autonomie locale et la politique nationale que se constitue le "raccrochage", le 
passage d'une politique jusqu'ici du logement et du social à une politique de la ville qui n'existe pas sans les élus. L'établissement d'une communication spécifique entre les élus, porteurs de la demande sociale, et l'État chargé d'adapter ses services à cette demande émerge dans ce cadre.

Les procédures connaissent un succès tel qu'entre le IXe Plan (1984-1988) et le Xe Plan (1989-1993), les contrats signés entre l'État et les villes passent de 148 à 400 sites classés "développement social des quartiers", soit 21 régions sur les $22^{6}$ que comptent la France. Cette inflation que nombre d'évaluateurs qualifièrent de "délirante" fut sans doute à l'origine d'un amoindrissement de son efficacité ${ }^{7}$. Parallèlement à la multiplication des contrats passés entre l'État et la commune dans les domaines de la famille, de l'éducation, de la prévention de la délinquance, de la formation professionnelle, on assiste à une évolution des dispositifs concernant la politique de la ville stricto sensu. Outre les contrats de développement social, dont la durée est en principe connexe au Plan, se crée la convention de quartier en direction des sites dont les difficultés ne sont pas encore jugées trop lourdes.

La méthode globale, qui a pour particularité de s'interroger sur l'espace d'intervention, préconise la mise en place de procédures à l'échelle de la commune, voire de l'agglomération. En effet, si le quartier cumule tous les handicaps, ce n'est peut-être pas en son sein que l'on trouvera la totalité des solutions aux problèmes posés. On assiste donc à l'élargissement du projet, tout au moins du discours : le développement social des quartiers n'atteindra ses objectifs que s'il est intégré au développement de la ville dans son ensemble. De quelques quartiers sensibles à intégrer à la ville, on passe à la ville dans son intégralité qui ne doit pas oublier dans son développement ses quartiers défavorisés. Cette évolution du raisonnement justifie la création des conventions ville-habitat, destinées à régir les opérations de réhabilitation de l'habitat, du pacte urbain de l'Arc nord-est qui couvre les villes en reconversion dans le nord et l'est de la France, et des contrats de ville lancés par la circulaire du 22 mai 1989 qui visent à approcher les problèmes urbains dans leur ensemble.

Quelle politique pour la ville?

En fait sous le terme politique de la ville, se profilent plusieurs approches complémentaires les unes des autres bien qu'en concurrence depuis maintenant plus de dix ans : les grands travaux (urbanisme et infrastructure), 1 'habitat (logement et cadre de vie), la citoyenneté (droits et devoirs de l'individu dans les domaines civil, politique, social et économique). La tension générée par une volonté de synthèse, qui n'est pas parvenue à réduire les logiques autonomes des différentes administrations intervenant dans le champ urbain, se retrouve dans les propos des plus hauts responsables de l'État $\left({ }^{8}\right)$, et traverse jusqu'à aujourd'hui la mise en œuvre de la politique de la ville. La réforme qui crée la Délégation interministérielle à la ville ${ }^{9}$ concrétise cette double ambition : fusionner les trois approches dans " le chantier de la ville ", répondre à l'attente immédiate des habitants des quartiers défavorisés en abordant la ville par les " cages d'escalier ". Le projet ainsi défini visait à unifier l'action de tout ce qui concerne la ville et ses habitants (soit $80 \%$ de la population française...), bien que ni l'école, ni la lutte contre le chômage ne soient expressément répertoriées dans les priorités de cette politique. Or, nombre d'études et de travaux de coopération internationale lancés par la Commission nationale de développement social des quartiers, poursuivis par la Délégation interministérielle à la ville, démontrent clairement qu'à la base des 
quartiers " sensibles ", on trouve la récession, le chômage et l'échec scolaire, avec une interdépendance des causes et des effets très marqués ${ }^{10}$. La volonté de rationaliser un ensemble mosaïque, produit de l'histoire, en renforçant une structure interministérielle aux dépens de ministères, dont certains connaissent une crise d'identité à la suite des partages de compétences issus des lois de décentralisation, a pu donner l'illusion d'une volonté politique forte. En fait, la réforme de 1988 marquera surtout une nouvelle étape dans l'institutionnalisation de la politique de la ville, renforcée au sein des différentes administrations et rendue plus cohérente par la réunion des trois objectifs jusque-là distincts : prévention de la délinquance, urbanisme, développement social des quartiers.

\section{Le retour de l'émeute urbaine}

Durant près d'une dizaine d'années, on a cru la crise des banlieues gérée, certain d'être passé à côté des situations de ghettos et de révoltes ethniques qu'affrontaient dans le même temps les plus grandes villes anglo-saxonnes. Vaulx-en-Velin, théâtre d'une émeute urbaine en dépit de réelles transformations dans le cadre des réhabilitations menées par la politique de développement social des quartiers, d'un renouveau associatif et d'un maire connu pour avoir fait siennes les inspirations majeures de la politique de la ville, a sonné le tocsin. Les événements d'octobre $1990^{11}$ qui se sont déroulés au Mas du Taureau ne peuvent être considérés comme des épiphénomènes ; les conditions du déclenchement des affrontements - la nouvelle de la mort de Thomas Claudio -, comme leur déroulement - mise à sac de commerces, heurts violenta avec la police, rodéos de voitures - s'inscriront par la suite dans un processus de répétition tragique que subiront la cité des Indes de Sartrouville en mars 1991, puis Mantes-laJolie en mai et juin de la même année.

11 Au-delà du constat d'échec trop rapidement effectué par nombre de commentateurs sur la politique de développement social des quartiers, au-delà du sentiment " d'injustice " ressenti par les élus qui mettent en avant l'accroissement constant des budgets municipaux consacrés à cette politique, les progrès réalisés en matière de cadre de vie dans les ZUP et les encouragements à la participation des habitants, il semble nécessaire de réfléchir aux conditions de production de cette violence, puis de s'interroger sur ce qu'elle révèle de la gestion des banlieues par le politique.

On partira des faits, tels qu'ils sont apparus à l'occasion des violences urbaines, puis on tentera d'en donner une explication de la façon la plus systématique possible en utilisant les éléments d'une enquête effectuée en mai et juin 1991 sur le dispositif de prévention de la délinquance de deux quartiers en développement social urbain. Ce travail a été effectué à la demande du maire de la ville et du préfet qui souhaitaient la visite d'observateurs extérieurs issus des administrations centrales (Intérieur, Éducation nationale, Justice, Délégation interministérielle de la ville et Conseil national des villes) pour émettre une opinion sur le fonctionnement du dispositif Cette démarche est intéressante à plus d'un titre. En effet, elle reflète l'inquiétude de nombreux élus locaux qui, suite aux événements de Vaulx-en-Velin, se sont tournés vers la puissance publique pour obtenir avis et recommandations, tant était partagé le sentiment que ce qui s'était produit dans l'agglomération lyonnaise était moins lié à une situation locale qu'à des tensions perçues dans l'ensemble des quartiers en difficulté. " On pensait tous qu'on était sur la bonne voie, qu'on sortait du tunnel. Le maire se désimpliquait. Puis, on est retombé dans le tunnel et le maire s'est réimpliqué. 
Depuis un an, on organise de très nombreuses réunions officielles de tous les élus et de tous les services. Mais dans les quartiers, la tension reste forte. J'ai le sentiment que les gens n'attendent plus rien, ils disent : vous faîtes ce que vous pouvez, mais... Aujourd'hui, beaucoup d'acteurs n'osent plus aller sur le terrain et sont démunis quand ils s'y trouvent. Moi, qui suis sur le quartier depuis 1979, je me suis récemment fait arracher ma sacoche dans la rue en plein jour, ce ne serait pas arrivé il y a cinq ans. Notre volonté c'est d'empêcher un événement grave qui laisserait des traces et serait difficile à rattraper " (adjoint au maire, conseiller général d'un quartier en développement social urbain) ${ }^{12}$. Ainsi, la violence urbaine a eu pour première conséquence de voir des maires, jusqu'ici soucieux de préserver leurs nouvelles prérogatives dans le champ urbain, interpeller les services de l'État, dont certains n'avaient pas renouvelé leur mode d'intervention en dépit des impératifs définis par la politique de la ville. C'est sans doute l'un des points les plus positifs des explosions de violence que d'avoir entraîné une rediscussion des interventions des différents partenaires dans les quartiers en difficulté mettant en évidence un certain nombre des dysfonctionnements des politiques sociales et urbaines transversales à l'origine de la politique de la ville.

Des émeutes par qui, pour quoi?

Les enquêtes menées dans les quartiers en difficulté permettent de mettre en évidence un premier paradoxe qui implique de poser la question de la présence policière au quotidien. Dans ces quartiers, tout se passe comme si l'on avait affaire à deux bandes rivales qui se côtoient constamment et finissent par s'affronter, faute de trouver d'autres interlocuteurs. Il semblerait que les rapports sociaux se soient progressivement réduits à un face à face entre les jeunes issus de l'immigration et les forces de l'ordre. Cette situation est liée à une évolution relativement récente qui révèle la dégradation de la qualité de vie dans les banlieues. Aujourd'hui, dans leur grande majorité les acteurs sociaux-professionnels qui travaillent dans ces quartiers n'y vivent plus ; éducateurs, responsables de l'office HLM, élus municipaux désertent la cité, dès la nuit tombée, pour un cadre plus paisible. " Je suis là jusqu'à 17 heures, mais le soir la cité m'est inconnue, on n'y rencontre pas les mêmes gens. Le soir, il n'y a plus personne, ni l'office HLM, ni la sauvegarde, ni les îlotiers. Un jour, j'avais oublié mes clefs, j'ai dû revenir la nuit, j'avais très peur " (un gérant d'HLM). A l'image des professionnels, les habitants du quartier le fuient à leur façon en se barricadant chez eux. Ainsi, le soir venu, les seuls interlocuteurs des jeunes sont les représentants de l'ordre, d'où l'impression que " les flics sont toujours derrière nous ", tandis que les habitants perçoivent leur cité comme désertée, abandonnée de tous, y compris de la police. Ce paradoxe entre des habitants en demande de présence sécuritaire, qui estiment que la police n'est plus là que de jour, pour régler les problèmes de circulation et arpenter les centres commerciaux, à qui l'on reproche de " surveiller les arbres ", et les jeunes, seuls " vrais " citoyens de ces quartiers, qui persistent à s'associer, à se mobiliser, à s'approprier l'espace ne serait-ce que par leur seule présence, pose problème.

Mais jusqu'ici, aucune interrogation d'ordre publique n'a trouvé sa place entre l'approche urbaine et l'approche sociale développée de façon parallèle, voire consécutive depuis maintenant dix ans. Les élus n'ont pas les moyens de contrôler les modes d'intervention de la présence policière, notamment ses rapports avec la jeunesse 
issue de l'immigration maghrébine, cible d'une injustice dont le harcèlement routinier des contrôles d'identité reste la forme la plus directe. Ils sont parallèlement confrontés à une demande sécuritaire qui réclame explicitement une augmentation de cette présence et risque de jouer l'agent provocateur de l'émeute par trop de contrôle systématique. Aujourd'hui, 1'explosion de la violence collective, dénonce, comme au temps des Minguettes, ces procédures policières routinisées. Cependant, s'y ajoute une dimension nouvelle ; la guerre du Golfe a certainement joué un rôle dans la mise en place d'un processus d'affrontement devenu régulier. Face aux suspicions très lourdes qui pesaient à son endroit (dans les quartiers, la population de souche achetait des armes, les lieux de culte musulmans étaient étroitement surveillés, les contrôles au faciès renforcés sous le nom " Opération vigipirate "), la population d'origine maghrébine a été sommée tout au long du conflit de choisir son camp. Le calme des banlieues a pu être interprété comme la démonstration d'une intégration enfin aboutie $^{13}$. Or, une fois le conflit terminé, la jeunesse issue de l'immigration en constatant qu'elle demeurait la cible privilégiée des contrôles systématiques se dotait de nouvelles références. La révolte palestinienne est devenue le modèle d'une Intifada des banlieues que l'on exerce à l'encontre de l'oppresseur désigné, la police, dans le cas français. " Depuis un an, on a eu 2 véhicules abîmés et 2 fonctionnaires blessés sur Valence le Haut, par jets de pierres. Ces jets sont de plus en plus gros. C'est très bien organisé : on nous appelle pour nous signaler un incident, on arrive, et on est attendu par des jeunes qui nous caillassent. Ce sont les majeurs qui montent le coup et les mineurs qui jettent les pierres parce que tout le monde sait que judiciairement parlant ils sont considérés comme irresponsables " (personnel de la brigade roulante).

La tension extrême entre jeunes des banlieues et forces de l'ordre constamment renvoyés à leur face à face trouve, de l'avis de nombreux acteurs de terrain, son explication dans le mode de fonctionnement du système judiciaire. Le discours récurrent est celui de l'impunité. La justice est souvent vilipendée, qui relâcherait les jeunes interpellés, de retour sur le quartier le jour même. Le sentiment d'impunité des mineurs est particulièrement mal ressenti par les forces de l'ordre, comme par les habitants du quartier, qui soulignent le vandalisme gratuit et la violence verbale pratiqués par des enfants de plus en plus jeunes. " Ceux de plus de 18 ans font faire des bêtises aux plus jeunes qui ne risquent rien. J'ai été cambriolé 10 fois en 20 ans, je n'y attachais pas tant d'importance, mais maintenant, ils cassent, ils brûlent, ça devient vraiment dangereux. La municipalité nous dit : si on vous casse quelque chose, on vous rembourse. Mais dans 10 ans, s'ils nous tuent, la municipalité nous dira : on vous enterre! " (commerçant).

17 Face à un sentiment d'insécurité croissante, de dégradation latente illustrée par les exemples quotidiens de cambriolages, de voitures fracturées, de vitrines cassées, le discours sécuritaire, très controversé au début des années quatre-vingt, émerge à nouveau : la prévention de la délinquance n'a pas suffisamment fait ses preuves, la solution c'est la répression, notamment pour un " noyau dur " que l'on cite dans les quartiers comme source de tous les maux sans jamais le chiffrer, le définir, le personnaliser - " Une quarantaine de personnes " (office HLM), " une cinquantaine de personnes " (brigade roulante), " dix à quinze personnes pourrissent l'atmosphère " (îlotiers), " notre liste est la même que celle de la police " (éducateurs)... Le noyau dur se définit soit par la délinquance multirécidiviste, soit par la toxicomanie ; il est avant tout le signe d'une situation objective qui crée cet état de marginalisation extrême qu'aucune structure ne prend en charge. Le noyau dur est considéré comme " perdu ", 
chaque intervenant estime qu'il n'entre plus dans sa sphère de compétence et que la police devrait in fine s'en charger, l'éloigner, le mettre à l'écart. " Les jeunes du noyau dur ont été suivis par tous les dispositifs, éducation, prévention, animation, on les connaît, ils sont une trentaine entre la drogue et la délinquance " (coordonnateur prévention de la délinquance). Ces discours traduisent sans doute une réalité vécue sur le terrain mais ils posent problème, entretenant l'illusion que de la " disparition " d'une trentaine de jeunes - " Si on arrête les caïds avant l'été, ça calme le jeu, ils passent l'été au frais " - dépend la quiétude retrouvée des quartiers.

Il s'agit ici de mettre en évidence certaines des observations que l'on peut faire sur les auteurs d'actes de délinquance et leur impunité dénoncée. En effet, dans les quartiers, l'une des assertions les plus souvent entendues est que la justice ne réprime pas suffisamment les personnes qui lui sont déférées, voire que les sanctions prononcées ne seraient pas exécutées. Il s'agit incontestablement d'un déficit de communication entre l'institution judiciaire locale et ses partenaires extérieurs. Il paraît en effet étonnant que les services de police puissent affirmer sans nuance que les auteurs de faits qu'ils interpellent sont immédiatement relâchés sur ordre du parquet, ou présentés au tribunal et tout autant remis en liberté sans qu'il y ait de suite pénale. Ces éléments d'appréciation, partagés par les élus et les habitants, relèvent davantage d'une méconnaissance des règles de procédures et de droit pénal que de véritables dysfonctionnements de l'institution judiciaire. I1 n'en demeure pas moins exact que le caractère systématique des délits mineurs (cambriolages, vols à la tire, dégradations...) pèse sur le quotidien des habitants et remet en cause la légitimité de l'institution policière ; en effet, ces affaires sont le plus souvent classées " sans suite ", non par une justice laxiste comme on l'entend dans les quartiers, mais par une justice à la recherche de réponses adéquates à ce type de délits. Par ce biais, elles contribuent à renforcer le sentiment d'une violence quotidienne vécue dans les quartiers qui préexiste à toutes les explosions de violence collective.

\section{La violence en bande?}

Une réflexion sur l'émeute urbaine ne peut être menée sans faire référence à la forte médiatisation actuelle du phénomène. En effet, les jeunes des banlieues sont de plus en plus explicitement désignés comme "violents en bandes". Or, cette violence répond davantage à des dysfonctionnements institutionnels qu'à l'émergence en France de bandes organisées.

21 En ce mois de novembre 1990, les manifestations des lycéens dans la capitale qui revendiquent "des profs, des moyens et la sécurité dans les établissements scolaires" s'achèvent sur des bris de vitrines, des vols à l'étalage, des voitures brûlées par dizaines et des affrontements avec la police. Les médias dénoncent l'irruption sur la place publique des nouveaux "casseurs", dont on souligne l'origine étrangère, le milieu défavorisé, et la domiciliation dans les banlieues Nord (Seine-Saint-Denis). Les pouvoirs publics décident la mise sur pied d'une enquête sur les auteurs des violences survenues à l'occasion de ces rassemblements ${ }^{14}$. Le travail sera mené à partir des procédures judiciaires en respectant le strict anonymat. II s'agissait d'une part de redonner une identité aux "casseurs" d'autre part de vérifier les "biens entendus" qui affirmaient qu'il s'agissait de bandes constituées de jeunes gens d'origine maghrébine ou africaine, enfin d'interroger l'idée selon laquelle "le Nord de Paris était venu en force". Sur les 46 interpellations réalisées, exceptées 3 ou 4 cas, il n'a pas été prouvé que les intéressés 
appartenaient à une bande organisée : ces bandes se sont formées spontanément dès les premiers débordements et se sont dissoutes aussitôt après. L'enquête démontre que les "casseurs" ne se différencient pas des lycéens manifestants en termes de nationalité, de lieux de résidence, d'âge ou de milieu social mais par contre s'en distinguent radicalement sur la question du cursus scolaire. La seule variable unifiante des interpellés est la situation d'échec scolaire généralisé dans laquelle ils se trouvent.

Ces violences ne traduisent-elles pas davantage le fait que, déjà exclus d'un système pour la réforme duquel les lycéens manifestaient ce jour-là, ces jeunes, en marge du collège comme de la manifestation, trouvaient en cassant et pillant la seule expression possible de leur malheur scolaire? A ce titre, les incidents d'origine criminelle de plus en plus fréquents dans les établissements scolaires des quartiers en difficulté peuvent appartenir à ce même registre, où l'on voit les exclus retourner la violence de ce vécu sur ceux-là même qui la leur font subir. La présence fréquente et répétitive, aux abords des collèges, de jeunes désœuvrés qui écoutent de la musique, injurient et menacent les élèves, instaure un climat d'insécurité vécu difficilement par le corps enseignant et les élèves. Ils peuvent, le cas échéant, montrer leur force de mobilisation lorsqu'un incident éclate. "Un jeune a frappé un professeur qui lui avait fait une remarque. J'ignore ce qui s'est passé mais en quelques instants j'avais une centaine de jeunes massés autour du lycée. Une montée de violence incompréhensible. J'ai dû fermer les grilles et sortir discuter avec les jeunes" (un responsable de collège). Cette attitude agressive révèle une attente vis-à-vis de l'institution scolaire, elle traduit l'ennui des jeunes en marge du système scolaire mais soucieux de ne pas s'en faire oublier radicalement. Le fait que les dégradations des établissements scolaires soient généralement pratiquées pendant les congés constitue en ce sens également une indication.

23 Ainsi, au terme bande, on préfèrera celui d'association épiphénoménale, liée à une rencontre, une occasion. Ces agrégations éphémères et peu structurées de jeunes peuvent se constituer lorsque l'événement se présente (manifestation, heurts avec la police, affrontement avec des jeunes d'une autre cité ${ }^{15}$ et ne se maintenir que le temps de l'explosion de violence. Il s'agit là de structurations territoriales qui doivent plus leur émergence à l'effet du hasard, des rencontres, du voisinage. Elles permettent aux jeunes de se reconnaître en quelque chose, de s'identifier en vase clos alors que le monde extérieur est le symbole de l'altérité. Cependant, on ne peut comparer ces rassemblements aux bandes telles qu'on les observent dans les grandes villes américaines, caractérisées par une activité délinquante organisée, une hiérarchie ritualisée, un territoire à défendre ou à conquériri ${ }^{16}$. Aujourd'hui dans les banlieues françaises, ce qui préexiste c'est l'ennui, l'absence de repères, le vide social, économique et culturel. L'anomie vécue, intériorisée, explique l'inexistence de véritables bandes, tout comme elle éclaire l'absence de projets scolaires, professionnels, mais aussi amoureux, des jeunes de ces quartiers. En ce sens, la "galère" décrite il y a quelques années par François Dubet ${ }^{17}$ reste le dénominateur commun d'une majorité des jeunes de banlieues.

La politique de la ville, dix ans après...

Trois tendances lourdes de la politique de la ville peuvent fournir des éléments d'explication. D'une part, l'essouff1ement de l'action publique sur le terrain. Les nouveaux dispositifs qui l'ont fondée, mission locale, prévention de la délinquance, 
développement social des quartiers, ont suscité un véritable enthousiasme de type militant chez ceux qui en avaient la charge mais ces derniers se sont épuisés à la tâche. Destinés à faire de l'accompagnement social, ils sont progressivement devenus des dispositifs centraux, seuls interlocuteurs d'une population touchée de façon privilégie par la crise économique. En effet, au début des années quatre-vingt, le gouvernement de gauche avait conçu ces dispositifs comme des structures de relais d'une "mauvaise passe" conjoncturelle que la reprise rendrait, à n'en pas douter, rapidement obsolètes. Or, plus de dix ans plus tard, la reprise n'est pas au rendez-vous, la situation sociale et économique des habitants de ces quartiers n'a cessé de se dégrader, mais ces dispositifs n'ont jamais reçu les moyens nécessaires pour accomplir un travail plus massif en direction d'une population en voie de paupérisation accélérée ${ }^{18}$ Outils mineurs pour une dégradation majeure, donc...

La seconde donnée d'importance réside dans l'incapacité de certaines administrations centrales à modifier leur mode de fonctionnement au regard des exigences de la procédure de développement social des quartiers. Les directions centrales des ministères de l'Intérieur ou de l'Education nationale ${ }^{19}$ ne sont pas réellement parvenues à s'investir dans les projets de réforme du début de la décennie quatre-vingt ; or les effets de la politique de la ville étaient largement soumis à cette capacité de réforme de l'administration et des services publics. Lorsque le maire de Vaulx-en-Velin, aux lendemains des incidents regrette que jamais ni la police, ni l'Education nationale n'aient participé aux rencontres réunissant les acteurs de la politique de développement social des quartiers, il désigne l'un des échecs majeurs de la politique de la ville : le partenariat, jugé nécessaire pour sortir des actions sectorialisées, n'a jamais pu trouver son inscription effective au niveau national comme sur le plan local. Dans les quartiers, que l'on parle de sa propre action, de ce qui est pratiqué ou de ce qui devrait être fait pour résoudre les problèmes, la vision que l'on donne des autres acteurs ou services concernés apparaît en règle générale négative ; elle oscille entre l'ignorance et l'accusation, lorsqu'elle ne mêle pas les deux genres : "La Justice ne suit pas" (police et préfecture), "L'Éducation nationale est le partenaire qui fait le plus défaut" (élu chargé de la prévention de la délinquance) tandis qu'un éducateur évoque l'inadaptation de la mission locale au quotidien des jeunes"... La politique de la ville a mis en évidence la difficulté pour le préfet dé fédérer les services extérieurs de l'État ; ainsi, le préfet n'a aucune autorité sur deux institutions clefs de cette politique, l'Education nationale, en la personne du recteur, la Justice en la personne du procureur général de la Cour d'appel. Les jeux de concurrence des acteurs et des administrations en charge de cette politique doivent être évoqués. Le développement des dispositifs en direction de la ville a créé de nouvelles fonctions, de nouveaux rôles dans l'espace socioprofessionnel, mais en a disqualifié d'autres ou du moins les remet gravement en cause. A la suite des émeutes du Mas du Taureau, le Conseil national des villes, dont l'un des deux vice-présidents est Gilbert Bonnemaison, initiateur de la prévention de la délinquance, avait annoncé la mise sur pied d'un audit public qui aurait réuni toutes les parties concernées (jeunes, habitants du quartier, police, acteurs professionnels de la politique de développement social urbain, élus municipaux, préfet...). Rendue publique, cette initiative avait été saluée comme une tentative de faire avancer le débat sur les conditions de la mise en œuvre de la politique de la ville. Elle fut annulée au dernier instant, sans aucune explication officielle. 

enregistré en ce qui concerne la participation des habitants. Cette participation, conçue comme le pilier de la politique de développement social des quartiers, n'a jamais émergé. A aucun moment, les habitants ne sont devenus des "acteurs de leur propre changement", pour reprendre la belle formule d'Hubert Dubedout. Parallèlement, le mouvement beur né de l'émeute des Minguettes, concrétisé dans la grande marche pour l'égalité de décembre 1983 à l'issue de laquelle le président François Mitterrand recevait une délégation de jeunes émeutiers devenus militants, s'est vu laminé par la récupération politique, l'énorme succès médiatique d'associations nationales (SOS Racisme, France Plus) qui reprenaient tous leurs thèmes de revendications et les multiples scissions internes ${ }^{20}$. Aujourd'hui, le mouvement associatif franco-maghrébin issu des banlieues n'est plus, sans doute également parce que les revendications ne sont plus du même type : des problèmes d'intégration culturelle on est passé aux difficultés d'intégration économique et sociale auxquelles sont confrontés tous les jeunes de banlieues issus d'un milieu défavorisé quelque soit leur origine ${ }^{21}$. Face à la multiplicité des exclusions subies, aux désillusions de l'action associative, les jeunes adoptent un discours violent qui remet en question l'ensemble des acteurs travaillant ou vivant sur les quartiers. La traduction première reste la dégradation quotidienne de l'environnement et des relations sociales. Mais, elle peut à l'occasion leur fournir le mobile d'une action collective où la violence devient l'expression du ressentiment vécu par tous. "Le problème des quartiers, c'est l'emploi. On voit beaucoup de chantiers, de constructions mais jamais les jeunes de chez nous n'y travaillent. On est tous en liste d'attente pour des stages et on attend l'été pour faire les fruits. C'est pas une vie. Nos petits frères, on se demande ce qu'ils vont devenir. Pourquoi y a-t-il des éducateurs ? Pourquoi pas des jeunes qui viendraient d'autres villes et qui nous diraient comment ça se passe chez eux ? Les éducateurs, ils arrivent dans le quartier à midi. On frappe chez eux, ils sont au bar. Si on veut monter un projet, rien que pour le taper, il faut demander à la mairie, qui nous envoie au centre social, qui nous dit d'aller acheter du papier. Les îlotiers, au début, on discutait avec eux, on a même fait des matchs de foot. Maintenant qu'ils nous ont tous fiché avec adresses et photos, on ne les voit plus. Ils profitent de leur métier pour nous maltraiter. Les médias nous salissent, ils nous traitent de voleurs. Les commerçants parlent trop, on les nourrit en achetant chez eux et eux ils disent aux médias qu'on est des dealers, alors on casse... " (propos tenus par une dizaine de jeunes).

Des effets contrastés de la violence dans les quartiers...

La violence urbaine exercée aujourd'hui dans les quartiers en difficulté pose problème par ce qu'elle exprime mais également par ce qu'elle déclenche. Elle semble amorcer un nouveau mode de relation entre les jeunes des cités et le monde politique. Ces violences, comprises comme l'expression d'un malaise collectif, sont entendues par les responsables institutionnels. C'est parce qu'il y a eu Vaulx-en-Velin que Michel Delebarre est devenu ministre de la Ville ${ }^{(22}$ ), ce sont les émeutes de Sartrouville qui ont obligé le maire Laurent Wetsel à débloquer la procédure de développement social urbain dont il conditionnait la mise en place à un renfort des effectifs de la police municipale. Ces réactions "immédiates" aux débordements créent les conditions d'une reproduction de ce type de violence, puisque désormais les jeunes ont acquis la certitude qu'il leur faut tout casser avant d'être écoutés. Cette culture de l'émeute 
risque d'être de plus en plus difficile à gérer parce qu'elle tend à menacer de façon permanente l'institution locale, et à travers elle les dispositifs nationaux. Désormais, en préalable de toute négociation, les jeunes menacent : "On fait sauter ta mairie si tu ne nous envoies pas en vacances" a-t-on entendu dans tous les services de prévention de la délinquance dans les quartiers. D'où la rumeur véhiculée ensuite par les acteurs de la politique de la ville, rejoints par les élus locaux : "l'été sera chaud". Ainsi, le développement d'une instrumentalisation de la violence urbaine est l'un des aspects les plus négatifs de ces explosions de colère : ì fait peser sur le quartier une atmosphère quotidiennement détestable ressentie par tous, habitants, jeunes, forces de l'ordre, travailleurs sociaux, élus locaux...

31 Ces violences ont éclairé les dysfonctionnements de la politique de la ville à plusieurs niveaux. Sur le plan local, on assiste sans doute à la prise de conscience de l'insuffisance d'une politique dont les principaux efforts ont porté sur les réhabilitations, à l'occasion desquelles on a tenté de plaquer quelques bons préceptes des initiateurs de la politique de la ville. La participation des habitants ces dix dernières années a davantage été sollicitée sur des options esthétiques sur le bâti que sur des questions qui auraient introduit une possible modification du cadre de vie. Cette participation n'a pas été possible parce qu'elle suppose de s'opposer, de proposer, et qu'elle implique de posséder des moyens culturels mais aussi techniques lorsque, par exemple, il s'agit d'argumenter face à des architectes-urbanistes. Jusqu'ici, l'ensemble des partenaires de la politique de la ville a pensé que les investissements sur le bâti auraient des conséquences décisives sur le malaise des banlieues, l'accompagnement social étant davantage perçu comme un "luxe". Aujourd'hui, on touche les limites extrêmes de cette réhabilitation pour laquelle la France a montré un grand savoir-faire. D'une part parce que ces travaux n'ont pas permis de résoudre le mal de vivre dans les cités ; alors que pour les professionnels, ils étaient perçus comme le moyen d'acquérir la paix sociale, ils étaient compris par les habitants comme leur étant dû : ne payent-ils pas ces modifications par des hausses de loyer qui les conduiraient bien souvent, si le choix leur en était donné, à les refuser? D'autre part, parce que les réhabilitations ont souvent été prétexte à l'expression d'un racisme exacerbé dans les quartiers où la population de souche française a le sentiment que ses "impôts vont aux Arabes". L'émeute urbaine a rappelé alors l'essence de tous les rapports qui fondent historiquement la politique de la ville : la population des quartiers défavorisés est toujours privée de la parole.

32 Au niveau national, les réponses aux manifestations de désespoir et de colère sont restées dans une logique politico-administrative - en France, lorsqu'on reconnait l'importance d'un problème on l'institutionnalise en créant un ministère, aux EtatsUnis, on met sur place un programme... - dont on ne peut pas encore mesurer les effets. L'explosion de violence dans l'agglomération lyonnaise en octobre 1990 a eu pour conséquence la mise en place d'une unité de commandement politique en la personne d'un ministre d'État, cinquième personnalité du gouvernement, expression d'un souci d'efficacité de l'action publique qui hiérarchise traditionnellement les objets de son travail. On espérait ainsi "obliger" certaines administrations centrales, dont on estimait qu'elles n'avaient pas su s'adapter aux nouvelles fonctions qui leur étaient imparties dans le cadre des politiques urbaines transversales, à s'engager davantage dans l'interministérialité et le partenariat. Dès sa nomination, le ministre de la Ville réclamait le renforcement de deux types d'actions : l'encadrement scolaire dans les quartiers en Zone d'éducation prioritaire, dont la géographie recouvre quasi- 
parfaitement les quartiers en développement social urbain, et la mise en place d'un système d'îlotage (police de proximité) dans un certain nombre de ces quartiers. Au sein de l'État, ces explosions ont donc déclenché un large débat sur les conditions de réussite d'une politique qui tendait à l'institutionnalisation la plus classique, oubliant que ce qui en avait fait son intérêt majeur était le principe d'innovation. A ce titre, de grands programmes d'évaluation au niveau territorial et national ont été mis en œuvre et financés par des fonds publics ${ }^{23}$. De même, le vide législatif qui caractérisait cette politique a été pour partie comblé par la loi Besson ${ }^{(24)}$, la loi sur la solidarité financière des communes ${ }^{25}$ et la loi d'orientation sur la ville ${ }^{26}$. Les débats sur les moyens financiers de la politique de la ville et leur utilisation relancés par l'explosion d'octobre 1990 et poursuivis tout au long de l'année 1991 à la suite de l'embrasement d'autres quartiers posent un certain nombre de problèmes quant à la coordination entre les effets d'annonce publique des décideurs nationaux et la gestion au niveau local pour les élus municipaux. Par exemple, l'annonce de crédits supplémentaires consacrés à la politique de la ville suscite aussitôt chez les jeunes des quartiers des demandes (salles des fêtes, terrain de sport, voyages...) dont ils ne comprennent pas qu'elles ne soient pas immédiatement satisfaites par le maire de leur ville mis dans l'impossibilité d'expliciter les délais de subventions. De même, l'idée selon laquelle les phénomènes de violence doivent être traités dans leur ampleur in situ en exerçant un effort particulièrement intensif sur une série de sites choisis en fonction d'handicaps particulièrement lourds (pourcentage de Rmistes, de chômeurs de longue durée, de familles monoparentales, d'immigrés, de logements sociaux, de familles issues des Dom-Tom...) permet l'établissement d'une liste de sites pilotes pour l'intégration sélectionnés au niveau de l'administration centrale (secrétariat général à l'Intégration, ministère des Affaires sociales responsable du Fonds d'action sociale) qui fait fi de toute la logique contractuelle entre l'État et les collectivités locales, fondatrice de la politique de la ville. Les élus locaux de la soixantaine de sites pilotes apprendront à l'occasion d'une conférence de presse nationale que leur commune bénéficiera d'une fraction des 200 millions supplémentaires alloués pour deux ans.

On se souvient que les émeutiers des Minguettes du début des années quatre-vingt étaient à l'origine du mouvement associatif des jeunes des banlieues issus de l'immigration. On constate, dix ans plus tard, que c'est toujours après l'épreuve de force que le mouvement associatif se constitue. Les événements d'octobre 1990 et de l'année 1991 n'ont pas démenti cette logique qui a vu, une fois l'explosion calmée, se constituer des comités d'action et de nouveaux leaders émerger. Des structures associatives, directement issues des incidents, à l'instar du comité Thomas Claudio devenu l'association Agora, très représentative de la jeunesse du Mas du Taureau, se sont construites à partir des incidents et avec une partie des fauteurs de troubles, acquérant une légitimité qui leur a permis de négocier avec les municipalités. Pour ces jeunes, il s'agit bien de renégocier au mieux leur entrée dans les rapports sociaux. C'est la violence des événements qui, paradoxalement, le leur permet. 


\section{NOTES}

1.Schwartz B., L'insertion professionnelle et sociale des jeunes (septembre 1981); Bonnemaison G., Face à la délinquance : prévention, répression, solidarité (décembre 1982) ; Dubedout H., Ensemble refaire la ville (janvier 1983), Publications de la Documentation française.

2.Ces politiques sociales territoriales ont aussi un passé : ainsi, de la procédure Habitat et vie sociale qui dès 1977 prône la nécessité d'un travail sur le bâti et sur le social préfigurant la politique de développement social des quartiers, ou du rapport Peyrefitte qui anticipent certaines des propositions de la commission Dubedout en incitant à la mise en place de coordinations départementales des services concourant à la prévention.

3.En 1986, les 3/4 des zones des établissements des zones de développement social des quartiers sont aussi en zones d'éducation prioritaires.

4.Une des lois principales de décentralisation est celle qui rend aux maires la décision du permis de construire et la production du plan d'occupation des sols permettant à la municipalité de contrôler son projet foncier, l'ensemble des ressources qu'elle recevait jusqu'ici lui étant désormais confié à travers la dotation globale d'équipement.

5.Jusqu'à la fin des années soixante-dix, les ingénieurs du corps des Ponts et Chaussées ont joué un rôle décisif dans la programmation de l'urbain. Thoenig Jean-Claude, L'ère des technocrates : le cas des Ponts et Chaussées, 1973.

6.Seule l'Auvergne n'a jamais participé à ce type de procédure.

7.La politique de la ville se caractérise essentiellement par la multiplicité de ses actions, la diversité des procédures, l'enchevêtrement des centres de décision. Le rapport Lévy, qui dressait en 1988 le bilan et les perspectives des contrats de plan de développement social des quartiers, insistait déjà sur les effets pervers d'une multiplication des contrats et des conventions : complexité d'un système difficile à manier pour l'ensemble des partenaires, dispersion des efforts financiers de l'État les rendant caduques...

8.Selon les circonstances, chacune de ces approches (bâti et accompagnement social contre habitants et citoyens) est appelée à la « rescousse ». A l'occasion de sa campagne électorale de 1988, le président de la République insistait sur la nécessité de « mieux organiser nos grands centres urbains » proposant que « les grands travaux du prochain septennat s'attaquent à ces problèmes et commencent à les résoudre ». Dès sa nomination, le Premier ministre Michel Rocard mettait l'accent sur les problèmes urbains, sur lesquels il revenait longuement dans sa déclaration de politique générale devant le Parlement, comme illustration de la « démocratie de tous les jours ».

9.Cette délégation préside au rassemblement et à la recomposition en son sein des trois structures préexistantes créées entre 1982 et 1983 (Commission nationale pour le développement social des quartiers, Conseil national de prévention de la délinquance, Banlieues 89).

10.Begag Azouz, " La révolte des lascars contre l'oubli à Vaulx-en-Velin ", Les annales de la recherche urbaine, $n^{\circ} 49-1989$, rappelle que dans la commune de Vaulx-en-Velin, $53 \%$ des habitants résident dans la ZUP, on compte plus de 40 nationalités, et près de $50 \%$ de moins de vingt ans ; $60 \%$ des élèves ont au moins un an de retard lorsqu'ils entrent en sixième et les moins de vingt-cinq ans connaissent un taux de chômage de 
plus de $35 \%$. Si $70 \%$ du budget communal est consacré à l'enfance et à la jeunesse à travers les activités de loisirs, de culture, de sport et d'éducation, le maire ne dispose d'aucun moyen pour réduire sensiblement le chômage.

11.Quatre-vingt millions de francs de dégâts matériels, tel est le bilan financier de trois nuits d'émeute.

12.Tous les dialogues rapportés dans le texte sont issus de cet audit qui a permis de rencontrer et de recueillir les témoignages des habitants, des jeunes, des commerçants, des gérants HLM, de personnes de l'institution policière, préfectorale, judiciaire, municipale, des opérateurs du développement social des quartiers.

13.Wihtol de Wenden Catherine, "Les Beurs et la guerre", Esprit - Les cahiers de l'Orient juin 1991.

14.Enquête réalisée par le Conseil national des villes : "Les casseurs : réflexions sur l'itinéraire social et familial de 46 éléments incontrôlés, interpellés à la manifestation lycéenne du 12 novembre 1990".

15.A l'origine des affrontements de novembre 1990 à Argenteuil (magasins dévalisés, heurts violents avec les vigiles d'une grande surface et la police), une rencontre prévue entre jeunes du val d'argent jeunes des Bosquets qui souhaitaient en découdre.

16.Jazouli Adil, Jeunes des banlieues, Violences et Intégration : le dilemme français, Etude exploratoire pour le Fonds d'action sociale, décembre 1990 ; du même auteur, Les années banlieues, Paris, Seuil, 1992.

17.Dubet François, La galère : jeunes en survie, Paris, éd. Fayard, 1987,

18.Les indicateurs de cette dégradation sont nombreux : le nombre des loyers impayés dans ces quartiers est en augmentation constante, de même que le nombre des chômeurs de longue durée et des jeunes de moins de 25 ans qui n'ont jamais travaillé.

19.Les conditions de la scolarité dans les banlieues restent aujourd'hui très préoccupantes : " $1 / 3$ des élèves en situation catastrophique en CM2 et $1 / 3$ d'exclus ou de baladeurs. Lorsqu'une institution travaille pour un tiers du pourcentage, il y a beaucoup de questions à se poser" (proviseur en région parisienne) - Les mouvements collectifs et la violence dans les villes - Commission du Conseil national des villes, présidée par Pierre Cardo, maire de Chanteloup les Vignes, juillet 1991.

20.Negrouche Nasser, "L'Echec des associations franco-maghrébines issues de l'immigration (1980-1990)", Esprit, janvier 1992.

21.Un jeune de moins de 25 ans sur 5 pointe à l'ANPE en 1990. Au 30/12/1990, les moins de 25 ans représentaient $27 \%$ du nombre total des demandeurs d'emplois, in Les mouvements collectifs et la violence dans les villes - Commission du Conseil national des villes, préside par Pierre Cardo, maire de Chanteloup les Vignes, juillet 1991. 22.A l'occasion des troisièmes assises de Banlieues 89 les 4 et 5 décembre 1990, soit deux mois à peine après les événements du Mas du Taureau, François Mitterrand annonce la création d'un ministère de la Ville en ces termes : "Il faut absolument qu'il y ait auprès du Premier ministre un membre du gouvernement, un ministre d'État ou un ministre tout simplement - peu importe son titre - dont le travail à la tête d'une équipe restreinte consistera à être l'animateur le pourfendeur, l'avocat, l'intendant permanent qui attirera l'attention d'hommes et de femmes qui, après tout, ne demandent pas mieux que de réussir cette grande aventure mais qui pensent à autre chose, qui ont d'autres soucis, d'autres compétences", in Pour en finir avec les grands ensembles, Banlieues 89, Assises de Bron, décembre 1990 (publication DIV). 
23. Comité d'évaluation de la politique de la ville (Délégation interministérielle à la ville), Programme d'observation et d'évaluation des politiques publiques dans les banlieues (Banlieuscopies).

24.Loi (janvier 1991j sur le logement des plus démunis.

25.Loi du 13 mai 1991 : par le moyen de la dotation générale de fonctionnement, une solidarité financière au profit des communes confrontées aux dysfonctionnements urbains les plus dommageables.

26.Loi du 13 juillet 1991 : fixe les principes et les moyens d'une évolution mieux maîtrisée et plus équilibrée des territoires urbains.

INDEX

Index géographique : France

Mots-clés : délinquance, répression, villes/ sociologie urbaine, crises politiques 